\title{
Viabilidade de adesão do curso de Odontologia da UFCG ao GraduaCEO
}

\author{
Kallyne Kennya Fernandes Alencar Furtado*, Winilya de Abreu Alves*, Luciana Ellen Dantas Costa***, \\ Renato Lopes de Sousa**, Rachel de Queiroz Ferreira Rodrigues***, Rodrigo Alves Ribeiro***, João \\ Nilton Lopes de Sousa****
}

* Estudante de graduação em Odontologia pela Universidade Federal de Campina Grande, UFCG Campus Patos

** Cirurgião-Dentista pela Universidade Federal da Paraíba, UFPB

*** Professores Doutores da Universidade Federal de Campina Grande, UFCG, Campus Patos

\begin{abstract}
RESUMO
O objetivo deste estudo foi avaliar a viabilidade da adesão do curso de Odontologia da UFCG ao programa GraduaCEO/Brasil Sorridente, comparando o número de procedimentos realizados nas clínicas de Odontologia nos anos de 2013 e 2014 com a produtividade exigida pelo programa para o Padrão I. Para isso, foram analisados 1.243 prontuários de todos os pacientes atendidos nos respectivos anos. Nos anos de 2013 e 2014 foram realizados 3.238 procedimentos (269,83 por mês) e 5.725 procedimentos (477,08 por mês) respectivamente. Ao comparar estes dados à produtividade preconizada pelo Padrão I do GraduaCEO, verificou-se que apenas os procedimentos de baixa complexidade (atenção básica), atingiram, em 2014, a meta preconizada pelo programa do Ministério da Saúde/Brasil. Assim, concluise que o curso de Odontologia da UFCG, cujo objetivo é formar profissionais generalistas, não tem suporte de laboratórios, equipamentos e quantidade suficiente de professores para implementar uma atenção odontológica especializada em suas clínicas, tornando inviável a adesão ao GraduaCEO, que determina metas para procedimentos especializados de Endodontia, Periodontia, Prótese e Cirurgia Oral. Descritores: Atenção Básica. Procedimentos. Saúde Bucal.
\end{abstract}




\section{INTRODUÇÃO}

A clínica integrada, que se tornou parte da estrutura curricular dos cursos de Odontologia no Brasil em 1970, surge como a união dos conhecimentos adquiridos pelo aluno durante todo o curso e a prática clínica que ditará sua conduta profissional, visando sempre à formação de profissionais generalistas ${ }^{1}$. São nessas clínicas que o futuro profissional aprende, exerce ações, assimila condutas e adquire hábitos, fazendo delas espaços privilegiados não só para o aprendizado de procedimentos técnicos, mas também para o exercício da sua reflexão ética ${ }^{2}$.

O último levantamento epidemiológico em saúde bucal realizado no Brasil, a nível nacional, revelou dados alarmantes: cerca de $18 \%$ das crianças de 12 anos nunca foram ao dentista, situação semelhante encontrada em todas as regiões do país. Além disso, o serviço público foi marcadamente o mais utilizado ${ }^{3}$. Nesse contexto, os cursos de Odontologia apresentam grande importância no que tange a melhorar o acesso da população à atenção em saúde bucal de forma gratuita e universal ${ }^{4}$.

A partir dos resultados do Levantamento de Condições de Saúde Bucal da População Brasileira - SB Brasil - em 2003, ficou evidente a necessidade da organização da média complexidade em Odontologia na efetivação do Sistema Único de Saúde (SUS). Os dados apontaram para a gravidade e a precocidade da perda dentária, para a desigualdade relacionada ao acesso aos serviços odontológicos e a existência de quase $56 \%$ de idosos completamente edêntulos. Assim, em 2006 foi criado os Centro de Especialidades Odontológicas (CEO $)^{5}$.

Mesmo com a criação dos CEOs, o Brasil ainda enfrenta graves problemas de acesso à atenção de média complexidade. Mais recentemente, por meio de convênio entre os Ministérios da Saúde e Educação, o governo federal criou, para fortalecer o programa já existente chamado de Brasil Sorridente, o GraduaCEO, que se constitui de uma série de ações e serviços de saúde bucal a serem desenvolvidas e prestadas pelos cursos de graduação em Odontologia.

Para aderir ao programa, os cursos de Odontologia têm que pactuar metas de procedimentos a serem realizados anualmente, de acordo com o padrão escolhido, com no mínimo $900,1.700,2.700$ e 4.100 procedimentos odontológicos ao mês para os padrões I, II, III e IV, respectivamente. Estes procedimentos devem ser realizados nas áreas da atenção básica, incluindo procedimentos de promoção e prevenção em saúde bucal e procedimentos clínicos de baixa complexidade, tais como restauração de dente decíduo e permanente, exodontia de dente permanente, raspagem supragengival e procedimentos especializados nas áreas da Endodontia, Periodontia, Cirurgia Oral, Estomatologia, Radiologia e Reabilitação Oral Protética ${ }^{6}$.

Desta forma, o presente estudo teve como objetivo realizar uma análise retrospectiva dos atendimentos realizados na Clínica Escola do curso de Odontologia da Universidade Fedral de Campina Grande (UFCG), Campus Patos, para a avaliar a viabilidade de adesão ao Padrão I do programa GraduaCEO - Brasil Sorridente.

\section{MATERIAIS E MÉTODOS}

Trata-se de uma pesquisa transversal de caráter retrospectivo realizado com prontuários dos pacientes atendidos na Clínica de Odontologia da UFCG. O estudo foi aprovado pelo Comitê de Ética em Pesquisa da UFCG, sob protocolo CAAE 47493315.4.0000.518.

A amostra foi constituída por todos os prontuários dos pacientes atendidos na Clínica Escola de Odontologia nos anos de 2013 e 2014. Esses dois anos letivos foram os escolhidos pois não houve greve, o que poderia acarretar vieses no estudo. Para coleta de dados, foi utilizado um formulário adaptado da versão disponibilizada pelo programa GraduaCEO ${ }^{6}$. Antes do início da pesquisa, foi realizado um estudo piloto com 100 prontuários para calibrar o pesquisador com relação aos critérios de exclusão e aos procedimen- 
tos executados pelos alunos em todas as clínicas odontológicas. Nos prontuários, cada procedimento executado foi avaliado e contabilizado por único pesquisador. Foram excluídos os procedimentos não contabilizados pelo programa (ortodontia interceptativa) e aqueles registrados de forma incompreensível.

Os atendimentos realizados em cada ano foram quantificados e, em seguida, foi calculada a média mensal de cada procedimento para comparar com as metas preconizadas pelo Padrão I, formando 3 grupos: Grupo GraduaCEO Padrão I; Grupo UFCG 2013 e Grupo UFCG 2014. Os dados coletados foram transferidos para um banco de dados criado no software SPSS e analisados por meio de estatística descritiva.

\section{RESULTADOS}

Foram analisados 1.243 prontuários de todos os pacientes atendidos no período estudado. No ano de 2013, foram realizados 3.238 procedimentos, com média de 269,83 ao mês e, em 2014, 5.725 procedimentos, com média mensal de 477,08. Na tabela 1, pode-se observar a comparação entre os procedimentos odontológicos realizados na UFCG nos anos de 2013 e 2014, e as quantidades de procedimentos mínimos exigido pelo Padrão I do programa GraduaCEO.

Em relação aos atendimentos referentes ao nível da atenção básica, a média de procedimentos resultantes de ações de promoção e prevenção no ano de 2013 e 2014 foram inferiores ao preconi- zado pelo Padrão I do programa GraduaCEO. Quando se compara a média de procedimentos clínicos, o grupo UFCG 2014 foi superior à média mínima exigida pelo programa, com exceção apenas dos procedimentos restauradores, que foi levemente inferior. No ano de 2013, todos os procedimentos ficaram abaixo do mínimo preconizado (tabela 2 ).

$\mathrm{Na}$ tabela 3 pode-se observar que houve aumento nos procedimentos das especialidades de Periodontia e Endodontia no ano de 2014 em relação ao ano anterior, porém tais valores ficaram bem abaixo da média mensal para o Padrão I do programa, como pode ser visto na tabela 3.

$\mathrm{Na}$ especialidade de Cirurgia Oral a meta mínima preconizada pelo GraduaCEO não foi atingida para nenhum procedimento. Não houve procedimentos de curetagem apical, apicectomia, excisão de cálculo de glândula salivar, que não foram incluídos na tabela 4.

A especialidade de Radiologia, no procedimento radiografia periapical e interproximal, ultrapassou a meta mínima do Padrão I do GraduaCEO; no entanto, apresentou-se inferior ao programa para os procedimentos de radiografia oclusal e panorâmica (tabela 5).

Com relação aos procedimentos de Reabilitação Oral Protética, também não foram realizados em quantidade suficiente para alcançar o mínimo de procedimentos preconizados pelo Padrão I do programa. Os resultados da média mensal de cada procedimento estão na tabela 6 .

Tabela 1- Comparação entre a quantidade de procedimentos realizados na UFCG nos anos de 2013 e 2014, com a quantidade exigida pelo programa GraduaCEO Padrão I, de acordo com o nível de complexidade da atenção.

\begin{tabular}{c|ccccc}
\hline \multirow{2}{*}{ Complexidade da atenção } & \multicolumn{2}{|c}{ UFCG 2013 } & \multicolumn{2}{c}{ UFCG 2014 } & GraduaCEO Padrão I \\
& Total & Média mensal & Total & Média mensal & Média mensal \\
\hline Atenção Básica & 1.423 & 118,58 & 3.026 & 252,16 & 250 \\
Atenção Especializada & 1.503 & 125,25 & 2.359 & 196,58 & 550 \\
Reabilitação Oral Protética & 312 & 26 & 340 & 28,33 & 100 \\
\hline
\end{tabular}


Tabela 2 - Comparação entre a média mensal de procedimentos de Baixa Complexidade/Atenção Básica realizados na UFCG nos anos de 2013 e 2014 com a quantidade exigida pelo programa GraduaCEO Padrão I.

\begin{tabular}{l|ccc}
\hline Procedimentos de baixa complexidade/atenção & \multicolumn{3}{|c}{ Média mensal de procedimentos } \\
básica & UFCG 2013 & UFCG 2014 & GraduaCEO Padrão I \\
\hline Promoção e prevenção & $\mathbf{2 6 , 7 4}$ & $\mathbf{4 3 , 5 8}$ & $\mathbf{6 0}$ \\
Aplicação de selante (por dente) & 0 & 3,5 & \\
Aplicação tópica de flúor (individual) & 24,08 & 36,08 & \\
Evidenciação de placa bacteriana & 2,66 & 04 & $\mathbf{6 0}$ \\
Procedimentos clínicos & $\mathbf{4 9 , 9 8}$ & $\mathbf{8 7 , 4 7}$ & \\
Selamento de cavidade dentária & 13,75 & 19,58 \\
Capeamento pulpar & 7,58 & 9,08 \\
Restauração de dente decíduo & 7,91 & 17,5 \\
Exodontia de dente decíduo & 5,33 & 11,9 & \\
Ajuste oclusal & 0 & 01 & \\
Pulpotomia dentária & 0 & 0,25 & \\
Raspagem subgengivais (por sextante) & 8,33 & 15,16 \\
Raspagem polimento supragengivais & 7,08 & 13 \\
Restaurações & $\mathbf{1 9 , 9 1}$ & $\mathbf{6 6 , 4}$ & $\mathbf{8 0}$ \\
De dente permanente posterior & 15,33 & 48,9 & \\
De dente permanente anterior & 4,58 & 17,5 \\
Exodontia de dente permanente & $\mathbf{2 1 , 9}$ & $\mathbf{5 4 , 6}$ & \\
\hline
\end{tabular}

Tabela 3 - Comparação entre a média mensal de procedimentos especializados de Endodontia e Periodontia realizados na UFCG nos anos de 2013 e 2014 com a quantidade exigida pelo programa GraduaCEO Padrão I.

\begin{tabular}{l|ccc}
\hline $\begin{array}{l}\text { Procedimentos de alta complexidade / atenção } \\
\text { especializada: Endodontia e Periodontia }\end{array}$ & \multicolumn{3}{|c}{ Média mensal de procedimentos } \\
UFCG 2013 & UFCG 2014 & GraduaCEO Padrão I \\
\hline Endodontia & 6,08 & 14,8 \\
Acesso à polpa e medicação (por dente) & 8 & 4,4 \\
Curativo de demora c/ ou s/ preparo & 0,41 & 0,58 & 0,08 \\
Obturação de dente decíduo & 0 & 0 & \\
Retratamento em dente permanente birradicular & 0 & 0,25 & $\mathbf{9 0}$ \\
Retratamento dente permanente de 3 ou + raízes & 0 & $\mathbf{2 0 , 1 1}$ & $\mathbf{1 5}$ \\
Retratamento dente permanente uni-radicular & $\mathbf{1 4 , 4 9}$ & 7,25 & 20 \\
Resultado consolidado & 3,41 & 0,5 & \\
Obturação dente permanente unirradicular & 1,5 & 0 & \\
Obturação dente permanente birradicular & 0 & 01 & \\
Obturação dente permanente com 3 ou + raízes & & 01 & $\mathbf{1 0 0}$ \\
Periodontia & 0,58 & 0,5 & $\mathbf{2 , 5}$ \\
Gengivectomia (por sextante) & 0,58 & \\
Gengivoplastia (por sextante) & 0,01 & $\mathbf{1 , 1 7}$ & \\
Tratamento cirúrgico periodontal (por sextante) & & \\
Resultado consolidado & &
\end{tabular}


Tabela 4 - Comparação entre a média mensal de procedimentos especializados de Cirurgia Oral realizados na UFCG nos anos de 2013 e 2014 com a quantidade exigida pelo programa GraduaCEO Padrão I.

\begin{tabular}{l|ccc}
\hline Procedimentos de alta complexidade / atenção & \multicolumn{3}{|c}{ Média mensal de procedimentos } \\
especializada: Cirurgia Oral & UFCG 2013 & UFCG 2014 & Gradua CEO Padrão I \\
\hline Remoção de dente retido (incluso / impactado) & 0,33 & 0,08 & $\mathbf{3 0}$ \\
Frenectomia & 0,16 & 1,6 & $\mathbf{1 5}$ \\
Excisão de rânula ou fenômeno de retenção & 0,08 & 0,16 & $\mathbf{1 0}$ \\
Exérese de cisto odontogênico e não- & 0,08 & 0 & $\mathbf{1 0}$ \\
Curetagem periapical & 0 & 0 & $\mathbf{1 0}$ \\
Ulotomia/ulectomia & 0,41 & 0,41 & $\mathbf{1 0}$ \\
Apicectomia com ou sem obturação retrógrada & 0 & 0 & $\mathbf{5}$ \\
Remoção de tórus e exostoses & 0,16 & 0,08 & $\mathbf{5}$ \\
Tratamento cirúrgico para tracionamento dental & 0,08 & 0 & $\mathbf{5}$ \\
Correção de irregularidades de rebordo alveolar & 0,58 & 0,08 & $\mathbf{4}$ \\
Aprofundamento de vestíbulo oral (por sextante) & 0 & 0 & $\mathbf{3}$ \\
Correção de bridas musculares & 0 & 0,25 & $\mathbf{3}$ \\
Outros procedimentos cirúrgicos & & & $\mathbf{8 0}$ \\
Drenagem de abcesso da boca e anexos & 0,08 & 0 & \\
Odontosecção/Radilectomia/Tunelização & 0,5 & 0 & 0 \\
Marsupialização de cistos e pseudocistos & 0,16 & 0 & \\
Reimplante e transplante dental (por elemento) & 0,25 & 0 & \\
Tratamento de alveolite & 0,08 & 0 & \\
\hline
\end{tabular}

Tabela 5 - Comparação entre a média mensal de procedimentos de Estomatologia e Radiologia realizados na UFCG nos anos de 2013 e 2014 com a quantidade exigida pelo programa GraduaCEO Padrão I.

\begin{tabular}{l|ccc}
\hline $\begin{array}{l}\text { Procedimentos de alta complexidade / atenção } \\
\text { especializada }\end{array}$ & \multicolumn{3}{|c}{ Média mensal de procedimentos } \\
\hline Radiologia e Imaginologia & $\mathbf{2 0 1 3}$ & UFCG 2014 & Gradua CEO Padrão I \\
Radiografia periapical e interproximal & 95,66 & 111,5 & $\mathbf{3 0}$ \\
Radiografia panorâmica & 0 & 0 & $\mathbf{3 0}$ \\
Radiografia oclusal & 0,75 & 1,08 & $\mathbf{2 0}$ \\
Estomatologia & $\mathbf{1 , 2 5}$ & $\mathbf{1 , 7 5}$ & $\mathbf{3 0}$ \\
Biópsia de glândula salivar & 0 & 0 & \\
Biópsia de osso do crânio e da face & 0 & 0 & \\
Biópsia dos tecidos moles da boca & 1,25 & 1,75 & \\
\hline
\end{tabular}


Tabela 6 - Comparação entre a média mensal de procedimentos de Reabilitação Oral Protética realizados na UFCG nos anos de 2013 e 2014, com a quantidade exigida pelo programa GraduaCEO Padrão I.

\begin{tabular}{l|ccc}
\hline Procedimentos de alta complexidade / atenção & \multicolumn{3}{|c}{ Média mensal de procedimentos } \\
especializada & UFCG 2013 & UFCG 2013 & Gradua CEO Padrão I \\
Reabilitação Oral Protética & 1 & 0 & $\mathbf{2 0}$ \\
Prótese total mandibular & 1,5 & 0,25 & $\mathbf{1 0}$ \\
Prótese total maxilar & 1,08 & 0,08 & $\mathbf{7 0}$ \\
Prótese parcial mandibular removível & 0,58 & 0,08 & $\mathbf{2 7 , 5 2}$ \\
Prótese maxilar removível & $\mathbf{2 1 , 8 1}$ & 13,58 \\
Outros Procedimentos Protéticos & 12 & 1,3 \\
Moldagem dento-gengival & 1,08 & 1,25 \\
Reembasamento e conserto de prótese & 0,5 & 4,5 \\
Cimentação de prótese dentária & 3,66 & 3,25 \\
Adaptação de prótese dentária & 3,66 & 0 \\
Instalação de prótese dentária & 0 & 0,41 \\
Coroa de aço e policarboxilato & 0 & 2,3 \\
Placa oclusal & 0,5 & 0,6 \\
Coroa provisória & 0,16 & 0,33 \\
Prótese temporária & 0,25 & \\
Prótese Coronárias / intra-radiculares fixas / & & \\
adesivas (por elemento) & & \\
\hline
\end{tabular}

\section{DISCUSSÃO}

O conhecimento das condições de saúde da população, por meio do uso da Epidemiologia, deve orientar a organização dos serviços, promovendo a adequação às suas reais necessidades ${ }^{7}$.Uma das formas de se avaliar os serviços de saúde e orientar possíveis adequações é conhecer e analisar periodicamente o serviço tendo como pressuposto a avaliação da eficiência, eficácia e efetividade das estruturas, processos e resultados relacionados ao risco, acesso e satisfação dos cidadãos frente aos serviços públicos de saúde na busca da resolubilidade e qualidade $^{8}$.

Dessa forma o presente estudo permitiu uma análise da produtividade clínica e das condições da clínica de Odontologia da UFCG para se avaliar a viabilidade de adesão ao Padrão I do GraduaCEO, o qual preconiza uma média de 900 procedimentos mensais, sendo que 250 são procedimentos clínicos da Atenção Básica, 550 de Atenção Especializada e 100 procedimentos de Reabilitação Oral Protética ${ }^{6}$.

A partir da análise comparativa, em relação aos atendimentos referentes ao nível da atenção básica, a média mensal de procedimentos resultantes de ações de promoção e prevenção no ano de 2013 foi de 26,75 e em 2014 de 43,58, que foram inferiores ao preconizado pelo Padrão I do programa, que estabelece uma meta de 60 procedimentos/mês. Quando se compara a média de procedimentos clínicos, o grupo UFCG 2014 foi superior à média mínima exigida pelo programa, com exceção apenas dos procedimentos restauradores, que foram levemente inferiores. Esse resultado corrobora com a literatura ${ }^{1,9}$, uma vez que o objetivo da disciplina de Clínica Integrada é a formação de profissionais generalis- 
tas com enfoque na Atenção Básica.

$\mathrm{Na}$ Atenção Especializada e Reabilitação Oral Protética, as médias mensais ficaram bem abaixo das médias preconizadas pelo programa. Esse déficit na Atenção Especializada é visto em vários estudos que analisam produtividade em Clínica Integrada ${ }^{8-12}$.

O aluno de graduação, ainda com pouca experiência clínica, requer muito mais atenção por parte dos professores do que aquele aluno que realiza um procedimento clínico de atenção básica. Além disso, quando este aluno realiza procedimento especializado, o risco de iatrogenia é iminente. Assim, para torná-lo apto à realização de procedimentos especializados será necessária maior carga horária em sala de aula, em laboratório e em clínica, o que inviabiliza a adesão ao programa de um curso de Odontologia jovem $(8$ anos), com apenas 21 docentes exercendo atividades clínicas, sem a existência de pósgraduação, como é o caso do curso de Odontologia da UFCG. Muitos procedimentos, por serem menos frequentes na prática clínica diária, não foram realizados nos anos estudados, a exemplo dos procedimentos de Cirurgia Oral, tais como apicectomia, curetagem apical e excisão de cálculo de glândula salivar.

Além das limitações apresentadas como impedimentos para alcançar as metas dos procedimentos especializados preconizados pelo programa GraduaCEO, a clínica de Odontologia da UFCG ainda possui outros problemas que inviabilizam esse tipo de atendimento, como a falta de um laboratório de prótese, com profissionais técnicos, e um quadro reduzido de professores por especialidade odontológica. Neste sentido, é necessário que o curso de Odontologia passe por um processo de reestruturação para tentar cumprir e se adequar as exigências do programa, como criação de cursos de extensão, aperfeiçoamentos, especializações e principalmente aumentar o quadro de professores especialistas em todas as áreas da Odontologia.

A dimensão das metas é um fator que pode inviabilizar adesão dos cursos de Odontologia ao programa, que deveria prezar pela a qualidade dos procedimentos executados e não por sistema de produção de procedimentos. É muito difícil exigir qualidade de procedimentos especializados, quando executados por alunos com pouca experiência e vivência clínica, não sendo possível aumentar a velocidade e tempo de atendimento clínico. O programa GraduaCEO deveria colaborar com os cursos de Odontologia e com a atenção em saúde bucal da população, estimulando a prestação do serviço odontológico, porém sem a necessidade de metas mensais. $\mathrm{O}$ curso poderia receber o incentivo financeiro de acordo com sua produção ambulatorial mensal.

A iniciativa de utilizar a estrutura dos cursos de Odontologia na saúde pública e, de certa forma custear os procedimentos executados, é uma alternativa importante inclusive para melhorar a qualidade dos cursos e a saúde bucal da população. No entanto, a ideia de utilizá-los como Centros de Especialidades Odontológicas é questionável, pois muitos procedimentos pactuados são apenas realizados em curso de aperfeiçoamentos e especialização, tais como endodontia de molares, cirurgias periodontais de enxertos, cirurgias de dentes inclusos, entre outros.

\section{CONCLUSÃO}

Ao comparar as metas do GraduaCEO com a produtividade mensal das clínicas de Odontologia da UFCG conclui-se que, sem a existência de cursos de pós-graduação e contratação de professores especialistas em todas as áreas da Odontologia, é inviável a adesão do curso ao programa.

\section{ABSTRACT \\ Feasibility of UFCG Dental School accession to the GraduaCEO}

The aim of this study was to evaluate the accession feasibility of UFCG to GraduaCEO/Brazil Sorridente program, comparing the number of procedures performed in the dental clinics during the years of 2013 and 2014 with the productivity required by the program to the Standard I. For this, 
1,243 dental records of all patients treated in the respective years were analyzed. During the years of 2013 and 2014, 3,238 procedures (269.83 per month) and 5,725 procedures (477.08 per month) were performed, respectively. By comparing this data to the productivity recommended by the Standard I of the GraduaCEO, it was found that only low-complexity procedures (primary health care) reached the target in 2014, as recommended by the Ministry of Health/Brazil program. Therefore, it is concluded that the undergraduate degree in Dentistry of UFCG, which aims to graduate generalist professionals, does not have support of laboratories, equipment and enough quantity of professors to implement a specialized dental care in their clinics, making impossible the accession to GraduaCEO, as it determines goals for specialized procedures in Endodontics, Periodontics, Prosthodontics and Oral Surgery.

Descriptors: Primary Health Care. Ambulatory Surgical Procedures. Oral Health.

\section{REFERÊNCIAS}

1. Arruda WB, Siviero M, Soares MS, Costa CG, Tortamano IP, et al. Clínica integrada: o desafio da integração multidisciplinar em odontologia. RFO UPF 2009;14(1): 51-5.

2. Gonçalves ER, Verdi MIM. Os problemas éticos no atendimento a pacientes na clínica odontológica de ensino. Ciênc Saúe Colet 2007; 12(3):755-64.

3. Brasil. Ministério da saúde. Secretaria de Atenção á Saúde. Departamento de Atenção Básica, Projeto SB Brasil 2010: Condições de saúde bucal da população brasileira 20092010: resultados principais. 2010, 48p.

4. Mialhe FL, Gonçalo CS, Carvalho LMS. Avaliação dos usuários sobre a qualidade do serviço odontológico prestado por graduandos do curso de Odontologia a FOP/ Unicamp. RFO UPF 2008; 13(1):19-24.

5. Brasil. Ministério da Saúde. Caderno de Atenção Básica No 17. 2008. 70p.

6. Ministério da Saúde (Brasil). Portaria Interministerial $\mathrm{N}^{\circ} 1.646$, de 05 de agosto de
2014. Institui o componente GraduaCEOBRASIL SORRIDENTE no âmbito da política nacional de saúde bucal. Diário Oficial da União 06 de ago. 2014.

7. Barros SG, Vianna MIP, Chaves SCL. Descentralização da saúde e utilização de serviços odontológicos em 11 municípios da Bahia. Rev Baiana Saúde Pública 2009; 23(3):372-87.

8. Reis SCGB, Santos LB, Leles CR. Clínica integrada de ensino odontológico: perfil dos usuários e necessidades odontológicas. ROBRAC 2011; 20(52): 46-51.

9. Ferreira NP, Dantas TS, Sena-Filho M, Rocha DG, et al. Clínica integrada e mudança curricular: desempenho clínico na perspectiva da integralidade. Pesqui Bras Odontopediatria Clín Integr 2012; 12(1):3339.

10. Milani PAP. Avaliação e produtividade da disciplina de clinica integrada no curso de odontologia da universidade de Tuiuti do Paraná. São Paulo. Dissertação [Mestrado em Clínica Integrada] Faculdade de Odontologia de São Paulo; 2003.

11. Poi WR, Lawall MA, Simonato LE, Giovanini EG, Panzarini SR, Pedrini D. et al. Onze anos de avaliação dos planos de tratamento e tratamentos realizados pela disciplina de clínica integrada, faculdade de odontologia de Araçatuba-UNESP. Pesqui Bras Odontopediatria Clín Integr 2006; 6(3:)237-42.

12. Reis SCGB. Perfil, produtividade e eficiência em clinica integrada de ensino odontológico. Goiânia. Tese [Doutorado em Ciências da Saúde] Universidade Federal de Goiás; 2011.

Correspondência para:

João Nilton Lopes de Sousa

e-mail: jnlopesodonto@gmail.com

Av. dos Universitários, S/N, Rodovia

Patos/Teixeira, km1, Jatobá

58700970 Patos, PB 